\title{
Comunicação comunitária para a promoção da saúde e da cultura.
}

\author{
Communitarian communication for health and culture promotion. \\ Comunicación comunitaria para la promocion de la salud y de la cultura.
}

Fernando Oliveira Paulino $^{1}$, Juliana Soares Mendes e Leyberson Lelis Pedrosa ${ }^{2}$

\begin{abstract}
RESUMO
O presente artigo possui o objetivo de relatar o processo e os resultados do desenvolvimento de ações do projeto e disciplina Comunicação Comunitária a partir de sua institucionalização como Projeto de Extensão de Ação Contínua, inscrito no Decanato de Extensão da UnB em 2007. No mesmo ano, Comunicação Comunitária obteve recursos para o desenvolvimento de atividades em Planaltina a partir dos Projetos "Saúde e Comunicação Comunitária", que contou com apoio do Ministério da Educação, e "Cultura, Saúde, Educação Ambiental e Comunicação", fomentado pelo Ministério da Cultura. A partir da análise de documentos e registros do projeto, pode-se afirmar que as oficinas com jovens de Planaltina possibilitaram a troca de experiências entre universitários e estudantes secundaristas da Região Administrativa, gerando conhecimento e valorização da diversidade. As oficinas elaboradas e executadas com e para os jovens de Planaltina, bem como as aulas de Comunicação Comunitária com a presença dos universitários, permitiram a promoção da interdisciplinaridade e a prática de conceitos aprendidos nos estudos conceituais, além de proporcionarem o desenvolvimento social, econômico e cultural local, através do envolvimento dos jovens em atividades de promoção do meio ambiente e da valorização da cultura e da saúde.
\end{abstract}

Palavras-chave: Comunicação Comunitária; Juventudes; Saúde e Cultura.

\section{ABSTRACT}

\footnotetext{
${ }^{1}$ Doutor em Comunicação e professor da Faculdade de Comunicação da Universidade de Brasília. paulino@unb.br. Coordenador do Projeto Comunicação Comunitária (www.unb.br/fac/comcom)

2 Bacharéis em Comunicação pela Faculdade de Comunicação da Universidade de Brasília e pesquisadores do Projeto Comunicação Comunitária. julianasmendes@gmail.com; leypedrosa@gmail.com
} 
This article intends to describe the Communitarian Communications Project and academic course, the processes and results through its institutionalization as a University of Brasilia Extension Project in 2007. In the same year, Communitarian Communications obtained funding for the development of activities in Planaltina-Federal District alongside the projects "Health and Communitarian Communications", supported by the Ministry of Education and "Culture, Health, Environmental Education and Communication", sponsored by the Ministry of Culture. From the analysis of the project documents and records, it can be stated that the workshops developed for young people in Planaltina made possible the exchange of experiences among university and high school students, creating knowledge and valuing differences. The actions led to the promotion of interdisciplinary research and the daily practice of concepts learned in the theoretical component of the Project. They also stimulated social, economic and political developments by encouraging young people to preserve the environment, enhance the culture and promote health.

KEYWORDS: Communitarian Communications; Youth; Health and Culture.

\section{RESUMEN}

Este artículo tiene el objetivo de describir los procesos y resultados del desarrollo de accikones del proyecto y asignatura "Comunicación Comunitaria", partiendo de su institucionalización como un Proyecto de Extensión de Acción Contínua, inscrito en el Decanato de Extensión de la Universidad de Brasilia en 2007. En ese año, "Comunicación Comunitaria" obtuvo recursos para el desarrollo de actividades en la ciudad de PlanaltinaDF en el contexto de los proyectos "Salud y Comunicación Comunitaria", que contó con el apoyo del Ministerio de Educación y "Cultura, Salud, Educación Ambiental y Comunicación", patrocinado por el Ministerio de la Cultura del Brasil. Del análisis de los documentos y registros del proyecto, se puede afirmar que los talleres para los jóvenes de Planaltina hicieron posible el intercambio de experiencias entre estudiantes universitarios y estudiantes de bachillerato (escuela secundaria), dee esa región, generando conocimiento y valoración de las diferencias. Los talleres elaborados e ejecutados con y para los jóvenes de Planaltina, así como las clases de Comunicação Comunitaria con la presencia de los universitarios, peremitieron la promoción de la interdisciplinaridad y la práctica de conceptos aprendidos en estudios teóricos. También proporcionaron estimuló al desarrollo social, económico y cultural local, por medio de la inclusión de los jóvenes en actividades de preservación del medio ambiente y de la valoración positiva de la cultura y de la salud.

PALABRAS CLAVE: Comunicación Comunitaria; Juventud; Salud y Cultura.

\section{INTRODUÇÃO}

A disciplina Comunicação Comunitária surgiu no primeiro semestre de 2002 na FAC-UnB para atender a demanda da recém criada Rádio Laboratório de Comunicação Comunitária (Ralacoco FM). Após um ano de atuação, por sugestão dos universitários que pretendiam continuar as atividades de ensino, pesquisa e extensão, criou-se a matéria Comunicação Comunitária 2. Desde o início das atividades, aproximadamente 600 universitários se 
matricularam nas disciplinas (fundamentalmente estudantes de Comunicação, Biblioteconomia, Serviço Social, Letras, Geografia, Engenharia Civil, Filosofia, Nutrição, História, Ciências Naturais e Agronegócios) que a partir de 2007 compõem o Projeto de Extensão de Ação Continuada Comunicação Comunitária (www.unb.br/fac/comcom).

Além de oficinas, seminários nas semanas de extensão da UnB, debates na TV Comunitária de Brasília e cursos com a presença de representantes de entidades governamentais e nãogovernamentais, o Projeto desenvolveu diversos produtos de mobilização social. Dentre este material, podemos citar:

- CD de spots de radio e rap "Proteja-se, use camisinha", com o apoio do Programa Nacional de DST-AIDS de da Gerência de DST e AIDS da Secretaria de Saúde do DF;

- CD de spots e rap "Aconteça o que acontecer, use camisinha”, com o apoio da Secretaria de Saúde do DF;

- CD de spots e rap "Tuberculose e Hanseníase têm cura, procure se informar", em parceria com a Escola Superior de Ciências da Saúde, com o Sindicato de Radialistas do DF, com a Fundação Damien e com o Programa de Controle da Tuberculose no DF;

- O curta-metragem "Um olhar sobre o Varjão" com o argumento criado pelos moradores do Varjão;

- Spots de divulgação do Abril Indígena com o apoio do Instituto de Estudos Sócioeconômicos;

- Spots de promoção da saúde, educação ambiental, direito à comunicação e cultura;

- Kit audiovisual "Trilhas Sociais" (www.trilhassociais.com)

O "Trilhas Sociais" resultou do apoio do Ministério da Cultura e do Ministério da Educação. O kit foi criado após mais de um ano de oficinas com jovens do ensino fundamental, ensino médio e universitários de Agronegócios e Ciências Naturais do campus da UnB em Planaltina. Durante este tempo, 75 jovens participaram de atividades de produção do material audiovisual. O "Trilhas Socias" é formado por um DVD com quatro programas de dez minutos sobre saúde, comunicação cultura e educação ambiental; e um curta-metragem sobre a trajetória de lideranças comunitárias na em Planaltina (localidade distante $40 \mathrm{~km}$ de Brasília-DF); dois CDs com spots para rádio e músicas para promoção da saúde, meio ambiente e cultura; e um CD ROM com o registro de todo o processo, contendo uma cartilha para atividades de comunicação comunitária. O vídeo com as lideranças comunitária locais, "Planaltina Organizada", pode ser acessado em: http://video.google.com/videoplay?docid=4923235594837566353\&ei=_or7SLXMKo6qrg Lk7dHsDg\&q=planaltina+organizada.

Inicialmente, a proposta da disciplina era realizar atividades de comunicação para promover a melhoria da qualidade de vida da comunidade do Varjão, localidade de baixa renda 
próxima ao plano piloto e que iniciava o processo do Fórum de Desenvolvimento Local Integrado e Sustentável (DLIS). A disciplina se focou na importância do desenvolvimento social da região, considerado como "a expansão das 'capacidades' [capabilities] das pessoas de levar o tipo de vida que elas valorizam - e com razão" (AMARTYA, 2000, p.32). No transcorrer do projeto, houve o contato com outras comunidades e, atualmente, o foco principal é o trabalho desenvolvido em Planaltina, localidade que possui um campus da UnB.

Os objetivos do Projeto Comunicação Comunitária podem ser estabelecidos como a consolidação de um espaço de visão alternativa do fazer da Comunicação, na perspectiva de se "obter uma formação crítica como base para una ação social transformadora"1 (TUCHO, 2006, p.95); estímulo da responsabilidade social dos universitários; ampliação do trabalho de democratização da Comunicação para comunidades que não possuem ou possuem reduzidas condições de produção mediática; elaboração de produtos de comunicação qualificados para movimentos sociais; e expansão, para estudantes de outras áreas, da possibilidade de pensar e usar a Comunicação para a mobilização social. O Projeto se fundamenta no conceito de que "mobilizar é convocar vontades para atuar em busca de um propósito comum, sob uma interpretação e um sentido também compartilhados" (TORO; WERNECK, 1996, p.26).

O Projeto Comunicação Comunitária pretende desempenhar papel relevante dentro da UnB, uma vez que explora os conhecimentos e habilidades dos estudantes universitários, estimulando o contato com a realidade de outras comunidades e movimentos sociais. Os pesquisadores preenchem outro papel de destaque para a continuidade das atividades da disciplina. Em sua maioria, são recém-formados que passaram pela disciplina como estudantes ou integrantes da Ralacoco. Com eles, surgem novas perspectivas a serem exploradas: comunicação e saúde, comunicação e cultura, comunicação e novas tecnologias, etc. Assim, a Comunicação Comunitária tem mais condições de se transformar em espaço de apropriação do semelhante e diferente, do novo e antigo, recriando novas identidades culturais e possibilidades recriando novas identidades culturais e possibilidade de transformação social. Segundo Martín Barbero (2002, p.148), a "construção de uma nova identidade cultural, de uma cultura urbana popular: modo de aglutinação de crenças e comportamentos, modo de ressentir os problemas coletivos".

Devido às dificuldades inerentes à ação de extensão, identificou-se a necessidade de aumentar os laços institucionais das disciplinas Comunicação Comunitária 1 e 2 com a universidade, visando à ampliação do apoio acadêmico. Em 2007, institui-se, então, o Projeto de Ação Contínua de Extensão. O novo status das atividades facilitou a obtenção de transporte, a participação dos estudantes e a procura de apoio por meio de editais. A presença efetiva dos estudantes se tornou ainda mais sustentável com a possibilidade de bolsas de extensão.

Em relação a editais públicos, o Projeto Comunicação Comunitária se inscreveu no "Proext 2007" do Ministério da Educação e no "Proext Cultura 2007" do Ministério da Cultura. Em ambos os casos, propostas foram aprovadas para desenvolver ações de promoção da saúde, meio ambiente e cultura por meio da comunicação a partir da obtenção de equipamentos e recursos necessários para as atividades. O Projeto "Saúde e Comunicação Comunitária", 
que contou com recursos do Ministério da Educação, visou o debate contínuo sobre formas de prevenção de doenças e promoção da saúde. Seu foco principal foi o tema da nutrição e a segurança alimentar. As atividades práticas das oficinas com estudantes de ensino fundamental, ensino médio e universitários relacionaram hábitos saudáveis a partir do suporte da comunicação. Os produtos finais do projeto compreendem CDs de música e spots de rádio e quatro programas de TV de 10 minutos sobre saúde e comunicação, além de um livro para relatar a experiência. O Projeto "Cultura, Saúde, Educação Ambiental e Comunicação" contou com o apoio do Ministério da Cultura e pretendeu fortalecer a inclusão e a mobilização social de jovens de Planaltina. $O$ objetivo principal foi gerar o diálogo com os jovens sobre saúde, educação ambiental e comunicação de modo a produzir kits audiovisuais sobre cultura, saúde e meio ambiente.

\section{REFERENCIAL TEÓRICO-METODOLÓGICO}

O presente artigo possui o objetivo de relatar o processo e os resultados do desenvolvimento de ações do projeto e disciplina Comunicação Comunitária a partir de sua institucionalização. A reflexão é oportuna, pois pode demarcar mudanças geradas por esta nova condição do Projeto. O relato da experiência de comunicação para mobilização social permite divulgar a outros grupos a metodologia do Projeto e das disciplinas vinculadas às atividades, o que pode estimular sua replicação e também as contribuições externas de outros coletivos que desenvolvam atividades similares. A avaliação, ainda, é fundamental para a readequação das ações a realidades dos jovens envolvidos nas oficinas.

A palavra comunicação origina do termo em latim: communico. O conceito latino significa pôr em comum, repartir, dividir, reunir, misturar e associar. Ou seja, comunicar ultrapassa a simples transmissão de idéias e se caracteriza como a troca entre grupos. Também é uma ação que conduz à valorização da diversidade, uma vez que propõe a mistura de vários distintos entre si, não se restringindo tão-somente à linguagem, mas se configurando como ação em direção ao outro, é a reunião e associação entre pessoas. Dessa maneira, comunicar-se é diálogo, como defendeu o educador Paulo Freire. Para Eugênio Bucci (2008) "comunicar é justamente isto: tecer o sentido comum é buscar pontes de entendimento. É dialogar”. A comunicação, então permite o diálogo a partir de estruturas horizontais para a construção de novas idéias. Assim sendo, comunicar não significa meramente informar ou repassar comunicados. Estudos (LEFREVE, 2007) comprovam que a informação sobre hábitos saudáveis e práticas de prevenção a doenças não é suficiente para influenciar mudanças de comportamentos. Faz-se necessário um processo proativo contínuo de cultura e comunicação que envolva as pessoas para que hábitos saudáveis sejam incorporados em suas vidas.

O direito à comunicação e o acesso à mídia são mecanismos para buscar a garantia da promoção da cultura, da educação ambiental e da saúde. O tema saúde é transversal, em oposição à mera negação da doença. Portanto, a saúde se refere à integração de diversos direitos que gerem o bem estar e qualidade de vida dos jovens. Segundo, a Carta de Ottawa (OMS, 2007): "a promoção da saúde demanda uma ação coordenada entre todas as partes envolvidas: governo, setor saúde e outros setores sociais e econômicos, organizações voluntárias e não-governamentais, autoridades locais, indústria e mídia”. Por outro lado, o conceito de educação ambiental "considera a necessidade de incluir os diversos aspectos da

Tempus - Actas de Saúde Coletiva, vol. 3, n.1, p. 56-63, jan./mar. 2009. 
realidade, dando condições aos educandos para se posicionarem a agir em busca de caminhos justos e solidários para os desafios do processo de construção, ocupação e transformação não só do mundo natural, mas de sua vertente social, cultural e ética" (MEC/SEF, 2002, p.8).

Assim sendo, a comunicação se oferece como um instrumento de diálogo para a construção de alternativas socioambientais para os desafios que a sociedade enfrenta devido ao uso que fez dos recursos naturais. A comunicação também se destaca pela possibilidade de promoção de direitos humanos e qualidade de vida a partir dos debates que promove em meio às comunidades e o estímulo à mobilização social em prol da sustentabilidade. Porém, ainda que existam diversas mídias alternativas devido ao desenvolvimento tecnológico, o marco regulatório brasileiro possui mecanismos limitados para garantir a liberdade e pluralismo da comunicação. De acordo com Rogério Christofoletti (2003), cerca de sete grupos familiares controlam $80 \%$ da informação veiculada pelos meios de comunicação e aproximadamente uma em cada quatro empresas de radiodifusão brasileira pertence a políticos. O cenário de grande concentração empresarial com baixo grau de diversidade tem consequiências negativas porque a mídia possui grande incidência na vida cotidiana dos brasileiros.

O cidadão comum dificilmente possui acesso aos meios e equipamentos necessários para produzir sua própria comunicação. Então, a comunicação livre e plural se limita, por vezes, a instituições de comunicação como rádios e tevês educativas, públicas, comunitárias, fanzines e páginas na internet. Enquanto isso, instituições privadas de comunicação são potencialmente influenciadas por interesses econômicos. As consequiências desse modelo refletem-se na cultura e identidade brasileira, pois a mídia acaba por divulgar e criar tendências na música, moda e arte. Dessa maneira, o desenvolvimento do Projeto Comunicação Comunitária pode estimular a educação para e pela comunicação. Afinal, a reedição deste projeto e sua readequação para a realidade dos jovens participantes buscam estimular a leitura crítica dos meios, algo que estimula a criação de novas formas de participação política e ocupação de espaços midiáticos. Afinal, dentre oito mil jovens entrevistados pelo Ibase e Instituto Pólis (2005), 28,1\% participam de alguma forma de associativismo (que compreende ONGs, grupos culturais, esportivos, religiosos, etc.). Ainda, 57\% desses jovens afirmaram interesse e disponibilidade em debater temas da juventude em encontros e oficinas.

Considerando os diversos aspectos da comunicação, dos direitos humanos e da juventude envolvida, optou-se por compreender a realidade a partir da subjetividade dos pesquisadores e extensionistas. A metodologia utilizada é a da observação participante, "técnica pela qual o pesquisador integra-se e participa na vida de um grupo para compreender-lhe o sentido de dentro" (LAVILLE; DIONE, 1999, p.178). A observação participante é também fundamentada pela análise de documentos produzidos para registro e avaliação do desenvolvimento pedagógico de oficinas de comunicação, saúde e cultura. Os documentos selecionados para a análise são o planejamento e cronograma das oficinas, as avaliações mensais em grupos das oficinas e os relatórios produzidos pelos voluntários e os bolsistas.

\section{DESENVOLVIMENTO}

Tempus - Actas de Saúde Coletiva, vol. 3, n.1, p. 56-63, jan./mar. 2009. 
Entre 2007 e junho de 2009, foram realizadas cinco avaliações mensais, três relatórios anuais (dois redigidos por estudantes e um pelo coordenador do projeto), uma oficina de relato da experiência com os participantes e o registro das atividades em diários de campo. Estes documentos revelam a demanda por avaliações constantes sobre as oficinas. A partir dos registros e da análise dos objetivos do projeto, pode-se afirmar que as atividades possibilitaram a troca de experiências entre universitários e jovens secundaristas de Planaltina, gerando conhecimento e valorização da diversidade. As oficinas elaboradas e executadas para e com os jovens de Planaltina, bem como as aulas de Comunicação Comunitária, permitiram a promoção da interdisciplinaridade e a prática de conceitos aprendidos nos estudos conceituais, além de proporcionarem o desenvolvimento social, econômico e cultural local por meio do estímulo aos jovens para preservação do meio ambiente e da valorização da cultura e da promoção da saúde.

A transversalidade entre os diversos temas, com as teorias e práticas de comunicação servindo como elemento agregador, possibilitou progresso pedagógico. Contudo, segundo os universitários, ainda há a necessidade de se explicitar ainda mais as inter-relações entre os diferentes temas a partir de atividades práticas das oficinas. Apesar de considerar que os objetivos principais das oficinas foram alcançados, os universitários identificaram limitações que diminuem a dinamicidade destas atividades. Dentre os desafios apontados, pode-se destacar a dificuldade de tempo de alguns membros em participar do planejamento das atividades, o que dificulta a previsão e consideração de possíveis problemas antes do sábado, dia em que as oficinas são realizadas. Ainda assim, com diferentes graus de comprometimento, os universitários foram protagonistas na elaboração coletiva das oficinas e divisão de tarefas.

Os esforços de promoção do diálogo com os jovens participantes da oficina resultaram em atividades para o aprendizado e desenvolvimento de novas habilidades, a produção e gravação de spots para rádios e programas de televisão, além de produtos de comunicação experimentais e criativos. A troca entre os oficineiros e participantes mais persistentes se consolidou e todos puderam se conhecer melhor, abrindo possibilidades de compreensão de realidades distintas. Esta característica se aprofundou com o convite, em 2008, para que estudantes de Ciências Naturais e Agronegócios do campus de Planaltina se envolvessem com as oficinas de comunicação. Participantes, que antes se apresentavam mais tímidos ficaram mais à vontade para se inserirem das atividades propostas e explorarem sua criatividade. Inclusive, alguns estudantes secundaristas que se inscreveram desinteressados pela oficina, sentiram-se mais atraídos pela possibilidade de participar e interagir. Em conversas, eles indicaram como ponto positivo o fato de poderem falar mais que os universitários da equipe do projeto durante as oficinas. Portanto, houve a construção de conhecimento referente à comunicação e produção de materiais audiovisuais.

Os universitários da equipe do Projeto também puderam aprender com o processo de planejamento e execução das oficinas, além do aprendizado gerado pelo intercâmbio com os estudantes de Planaltina. A interação também gerou partilha do conhecimento entre os próprios estudantes da equipe, e entre os estudantes da equipe e a comunidade de Planaltina. Também acrescentou muito às oficinas a presença de estudantes de outras unidades acadêmicas da UnB (tais como Serviço Social e Nutrição). Para os universitários que participaram das reuniões de avaliação, um dos pontos mais positivos foi a 
participação, em um sábado, de um deficiente auditivo do CEM 2, que ensinou como lidar com a diversidade e também outras formas de comunicação.

Com a consolidação das atividades em 2008, os universitários reforçaram as atividades práticas e tentaram constantemente exibir os produtos que resultaram das oficinas, com o objetivo de atrair os jovens. Um elemento importante do desenvolvimento pedagógico foi a troca de experiências e companheirismo entre universitários e jovens das comunidades atendidas. Afinal, a comunicação e o aprendizado não se limitam à transmissão de informação, mas ambos requerem a vivência da realidade. Como enfatiza Paulo Freire (1977, p.67), "na comunicação, não há sujeitos passivos. Os sujeitos co-intencionados ao objeto de seu pensar se comunicam seu conteúdo". Outros fatores essenciais das oficinas foram: o reconhecimento e identificação entre os jovens de Planaltina e do Varjão (que participaram da produção dos vídeos e material sonoro), além do aprendizado e respeito às diferenças.

Para o futuro, há a necessidade de manter avaliação permanente dentro do cronograma das oficinas. Os participantes das atividades devem ser ouvidos para adequar as oficinas às suas demandas. Ainda, o registro destas análises e avaliações é fundamental para gerar o aprendizado não somente dos estudantes envolvidos, mas do Projeto Comunicação Comunitária, que prevê a matrícula de novos universitários a cada semestre e sua participação nas ações desenvolvidas na comunidade de Planaltina.

\section{BIBLIOGRAFIAS}

BARBERO, J.M. Ofício de cartógrafo: travessias latino-americanas da comunicação na cultura. Trad. Fidelina González. São Paulo: Edições Loyola, 2002.

BUCCI, E. Comunicação é diálogo. In: Jornal da Ciência, SBPC, abril, 2008. Disponível em: <www.jornaldaciencia.org.br>. Acesso em: 5 maio, 2008.

CHRISTOFOLETTI, R. Dez impasses para uma efetiva crítica da mídia no Brasil. Trabalho apresentado no Núcleo de Jornalismo. XXVI Congresso Científico Anual de Comunicação. Belo Horizonte/MG, Set. 2 e 6, 2003.

FREIRE, P. Extensão ou comunicação? Rio de Janeiro: Paz e Terra, 1977.

Juventude brasileira e democracia: participação, esferas e políticas públicas. São Paulo: IBASE e Instituto Polis, 2005.

LAVILLE, C.; DIONE, J. A construção do saber: manual de metodologias de pesquisa em ciências humanas. Trad. Heloísa Monteiro e Francisco Settineri. Porto Alegre: Editora Artes Médicas Sul Ltda.; Belo Horizonte: Editora UFMG, 1999.

LEFEVRE et al. Representações sobre dengue, seu vetor e ações de controle por moradores do Município de São Sebastião, Litoral Norte do Estado de São Paulo. Brasil. Cad. Saúde Pública. Rio de Janeiro, 23(7):1696-1706, jul, 2007. 
MEC/SEF. Políticas de melhoria da qualidade da educação: um balanço institucional. Brasília, $2002 . \quad$ Disponível em: $<$ http://portal.mec.gov.br/secad/arquivos/pdf/educacaoambiental/politicas.pdf>. Acesso em: 29 jul. de 2007.

OMS. Carta de intenções da Primeira Conferência Internacional sobre Promoção da Saúde. Ottawa - $\quad$ Canadá, $1986 . \quad$ Disponível em: <http://www.opas.org.br/coletiva/uploadArq/Ottawa.pdf>. Acesso em: 14 mai. 2007

SEN, A. Desenvolvimento como liberdade. São Paulo: Companhia das Letras, 2000.

TORO, B. Comunicação e Mobilização Social. Brasília: UnB, 1997

TORO, J. B.; WERNECK, N.M.D. Mobilização Social: um modo de construir a democracia e a participação. Brasil: UNICEF, 1996. Disponível em: $<$ http://www.projetoterrazul.hpg.ig.com.br/livro.pdf>. Acesso em: 25 jun. 2007

TUCHO, F. Educación en Comunicación: una introducción. Revista de Economía Política de las Tecnologías de la Información y Comunicación. Vol. VIII, n. 3, sep - dic. 2006. Disponível em: <http://www.eptic.com.br>. Acesso em: 27 fev. 2007.

1 Tradução dos autores. 